\title{
Human immunodeficiency virus-1 subtypes and antiretroviral drug resistance profiles among drug-naïve Brazilian blood donors
}

\author{
José Antonio Sá-Ferreira, Patrícia Alvarez Brindeiro, Saada Chequer-Fernandez, Amilcar Tanuri, and \\ Mariza Gonçalves Morgado
}

BACKGROUND: Human immunodeficiency virus-1 (HIV1) genetic variability and its implication on the development of new reagents were investigated by correlating time of infection and resistance to antiretroviral drugs.

STUDY DESIGN AND METHODS: Seventy-four plasma samples from Brazilian drug-naïve blood donors were assayed to further develop a panel of biologic reagents to be used in serology and molecular tests. After viral RNA extraction, cDNA was generated and used in nested polymerase chain reaction protocols with primers for the ENV (C2-V3 and gp41), protease (PR), and reverse transcriptase (RT) of HIV-1. Genomic sequencing was conducted to define HIV-1 subtypes and drug resistance mutations.

RESULTS: Subtype B was found in 83.8 percent of all samples, subtype $F$ in 2.7 percent, and BF mosaics in 11 percent of the cases. $B$ and $F$ different genetic profiles were evidenced: $B_{P R} B_{R T} B_{g p 120} B_{g p 41}, F_{P R} F_{R T} F_{g p 120} F_{g p 41}$, $\mathrm{F}_{\mathrm{PR}} \mathrm{F}_{\mathrm{RT}} \mathrm{B}_{\mathrm{gp} 120} \mathrm{~B}_{\mathrm{gp} 41}, \mathrm{~F}_{\mathrm{PR}} \mathrm{B}_{\mathrm{RT}} \mathrm{B}_{\mathrm{gp} 120} \mathrm{~B}_{\mathrm{gp} 41}$, and $\mathrm{B}_{\mathrm{PR}} \mathrm{B}_{\mathrm{RT}} \mathrm{F}_{\mathrm{gp} 120} \mathrm{~B}_{\mathrm{gp} 41}$. A single sample (1.4\%) was characterized as a $B C$ mosaic $\left(\mathrm{B}_{\mathrm{PR}} \mathrm{C}_{\mathrm{RT}} \mathrm{U}_{\mathrm{gp} 120} \mathrm{C}_{\mathrm{gp} 41}\right)$ and an $\mathrm{AGH}$ mosaic $\left(A G_{p R} G_{R T} H_{g p 120} H_{g p 41}\right)$ was first observed in the country. Antiretroviral resistance to nucleoside RT inhibitor was observed in one sample (1.35\%) showing M41L and T215S mutations. Nonnucleoside RT inhibitor and major PR inhibitor resistance mutations were not observed. Previously unseen patterns of resistance to T20 were found among HIV-1-infected drug-naïve individuals in Brazil. Recent infections were characterized in 21.6 percent of the blood donor samples included in this study.

CONCLUSIONS: The availability of characterized plasma samples and HIV isolates will allow the development of biologic reagents necessary to implement quality control programs and to develop, validate, and evaluate the performance of diagnostic kits, considering all HIV-1-circulating variants in the country.
$\mathrm{H}$ uman immunodeficiency virus-1 (HIV-1) is classified into three major groups: $\mathrm{M}$ (main), $\mathrm{O}$ (outlier), and N (new). Group M, responsible for the world AIDS pandemics, includes 9 subtypes, 3 subsubtypes and at least 29 circulating recombinant forms (CRFs) ${ }^{1-4}$ According to the National STD and AIDS Programme, from the beginning of the epidemics until June 2005, more than 371,000 cases of HIV-1 infection had been reported in Brazil. ${ }^{5}$ At least five different HIV-1 subtypes are cocirculating in the country: A, B, C, D, and $\mathrm{F}^{6-18} \mathrm{HIV}-1$ subtype $\mathrm{B}$ and the Brazilian variant $\mathrm{B}^{\prime}$ are the most frequent identified clades. ${ }^{6,19,20}$ Various BF and BC recombinant genomes were described, ${ }^{14,18,21}$ and CRF genotypes (CRF 02_AG, CRF 28_BF, and CRF 29_BF) have also been detected. ${ }^{4,18,22}$ Although the screening of blood supply is mandatory in Brazil, transfusion-transmitted HIV

ABBREVIATIONS: CRF(s) = circulating recombinant form(s); NNRTI = nonnucleoside reverse transcriptase inhibitor; NRTI = nucleoside reverse transcriptase inhibitor; $\mathrm{PI}(\mathrm{s})=$ protease inhibitor(s); $\mathrm{PR}=$ protease.

From the Section of Molecular Diagnostics, Diagnostic Technology Laboratory, Bio-Manguinhos, and the Laboratory of AIDS and Molecular Immunology, Department of Immunology, Oswaldo Cruz Foundation, Rio de Janeiro; and the Laboratory of Molecular Animal Virology, Departament of Genetics, Federal University of Rio de Janeiro, Rio de Janeiro, Brazil.

Address reprint requests to: Mariza Gonçalves Morgado, Laboratório de AIDS e Imunologia Molecular, Instituto Oswaldo Cruz-Fundação Oswaldo Cruz, Avenida Brazil, 4365-

Manguinhos, 21045-900-Rio de Janeiro, Brazil; e-mail:

mmorgado@ioc.fiocruz.br.

This work was partially supported by the Brazilian Research Council CNPq/RHAE Program.

Received for publication April 6, 2006; revision received June

14, 2006, and accepted June 24, 2006.

doi: 10.1111/j.1537-2995.2007.01069.x

TRANSFUSION 2007;47:97-102. 
infections still contribute with 0.15 percent of the annual reported AIDS cases as seen in the year 2005, with 17 new cases associated with blood transmission in the country. ${ }^{5}$ For that reason a continuous surveillance of HIV-1 genetic variability must be considered, given its implication over the capacity of screening tests to accurately distinguish HIV-1 groups and subtypes. ${ }^{23-25}$

Studies concerning the prevalence of HIV-1 subtype diversity among blood donors in Brazil are few and restricted to specific regions. The circulation of several (B, C, D, F, BF) HIV-1 subtypes and recombinant forms, however, has been reported. ${ }^{10,11,26-28}$

The percentages of primary drug resistance in reverse transcriptase (RT) and protease (PR) genomic regions vary greatly in different parts of the world, ranging from 3.4 to 12.4 percent in the United States, ${ }^{29} 10$ to 17 percent in France, ${ }^{30,31} 13$ percent in Germany, ${ }^{32}$ and 23 to 26 percent in Spain..$^{33}$ Lack of resistance mutations in Greek and Chilean drug-naïve individuals was reported, however, despite the high prevalence of sequence polymorphisms in both RT and PR regions. ${ }^{34,35}$ In a global study, performed in 2005 , it was demonstrated that the majority of mutations observed in subtype B viruses did not differ from those in non-B subtypes. ${ }^{36}$

Low prevalence levels of mutations associated to both nucleoside (NRTI) and nonnucleoside (NNRTI) RT and PR inhibitors (PIs) were reported in drug-naïve seropositive individuals in surveys conducted from 1999 through 2003 in Brazil. ${ }^{10,16,26,28}$

A new class of antiretroviral drug that targets virus fusion, comprising T20 (enfuvirtide), was developed and has been available in the market since 2003. Little and controversial information is available concerning the development of T20 resistance. Primary mutations associated to T20 resistance are distributed along the 36- to 45sequence residues of gp $41 .{ }^{37}$ The analysis of 95 samples from B and non-B T20 drug-naïve patients revealed no resistance mutations to this inhibitor. ${ }^{38}$ Besides, other authors ${ }^{39}$ have reported the occurrence of such mutations in 200 patients under monotherapy with T20 or highly active antiretroviral therapy. The presence of polymorphisms not associated to fusion inhibitor resistance has also been studied and its occurrence among B and non-B subtypes was evaluated..$^{39,40}$

In this work, we aimed at characterizing HIV-1 subtypes and recombinant genomes by nucleotide sequencing of the envelope (gp120 and gp41), RT, and PR regions. We also intended to evaluate the frequency of resistance mutations associated to PR, RT, and fusion (T20) antiretroviral drug inhibitors as well as the estimated time of HIV-1 infection. Considering the impact of viral diversity on the correct and adequate diagnosis and monitoring of the HIV-1 infection, we used plasma samples from drugnaïve HIV-1-infected blood donors living in different Brazilian geographic regions.

\section{MATERIALS AND METHODS}

\section{Samples}

Seventy-four discarded blood units were received during the years 2000, 2001, and 2004 from the routine of blood banks located in different Brazilian geographic regions, as follows: 6 from $\mathrm{N}$ (Amazon basin), 24 from NE (Bahia and Pernambuco states), and 44 from SE (Rio de Janeiro, Minas Gerais and São Paulo states). Samples, assayed in an anonymous unlinked study, were received at BioManguinhos, FIOCRUZ, Rio de Janeiro, Brazil, and retested by one rapid test (HIV-1 and -2, Bio-Manguinhos rapid test) and two enzyme immunoassays (EIAs; Vironostika HIV Uni-Form II antigen-antibody, Organon Teknika; and VIDAS HIV DUO, bioMérieux, Durham, NC). Confirmation of HIV-1 reactivity was performed by indirect immunofluorescence assay.

\section{Genetic analysis}

Viral RNA was extracted from all samples with a viral RNA mini spin kit (QIAamp, Qiagen Inc., Chatsworth, CA) according to the manufacturer's protocol. In the next step, complementary DNA (cDNA) was generated following the use of random primers and retrotranscription with RNase RT enzyme (SuperScript II, Invitrogen Corp., Carlsbad, CA). cDNA was used in nested polymerase chain reaction protocols with primers for the ENV C2V3 region of gp120, ENV gp41 immunodominant region, PR, and RT regions as previously described. ${ }^{10,16}$ Genomic sequencing was then conducted in an automated sequencer (ABI Model 3100, Applied Biosystems Perkin Elmer Cetus, Norwalk, CT) to define HIV-1 subtypes and to assess drug resistance based on ENV (gp41), RT, and PR genes. For subtyping analysis, samples were aligned based on software against a reference sequence set from the Los Alamos database (Clustal X 1.81 and Mega Version 3.0, http://hiv-web. lanl.gov/content/hiv-db/SUBTYPE_REF/align.html) and clustered phylogenetically by the neighbor-joining distance method with estimated distances by Kimura twoparameter method. Drug resistance mutation analysis of RT and PR genes were based on the Web sites http:// hivdb.stanford.edu/pages/algs/HIVdb.html and http:// www.iasusa.org/resistance_mutations/index.html. Drug resistance mutation analysis of ENV (gp41) to the fusion inhibitor, T20, was performed by comparing our results with those already described ${ }^{38-40}$ and also taking into consideration the variation observed in the 36- to 43amino-acid residue sequence of gp41 as indicated by http://www.iasusa.org.

\section{Identification of recent infected donors}

The discrimination between recent (less than 153 days) and long-term HIV-1 infection was estimated by a quantitative antibody assay, HIV-1 BED incidence CEIA 
(Calypte Biomedical Corp., Lake Oswego, OR), for the determination of the proportion of HIV-1-specific IgG with respect to total IgG present in the sample. The procedure is based on the serologic reactivity with gp41 peptides from distinct HIV-1 subtypes (B, E, and D), considering an optical density (OD) ratio of 0.8 as the cutoff. ${ }^{41}$

\section{RESULTS}

We have analyzed 74 plasma samples from HIV-1 drugnaïve Brazilian blood donors targeting the ENV (gp120 and gp41), RT, and PR HIV-1 genomic regions. In relation to subtype characterization, 83.8 percent $(n=62)$ of the samples presented virus that belong to subtype $B$ and 2 percent $(n=2)$ to subtype $F$, and BF mosaic structures were found in 11 percent $(n=8)$ of the isolates. The remaining two samples were comprised by one $\mathrm{BC}$ mosaic $\left(\mathrm{B}_{\mathrm{PR}} \mathrm{C}_{\mathrm{RT}} \mathrm{U}_{\mathrm{gp} 120} \mathrm{C}_{\mathrm{gp} 41}\right)$ and one $\mathrm{AGH}\left(\mathrm{AG}_{\mathrm{PR}} \mathrm{G}_{\mathrm{RT}} \mathrm{H}_{\mathrm{gp} 120} \mathrm{H}_{\mathrm{gp} 41}\right)$ recombinant sample, first described in the country. Various genotype profiles were observed for $\mathrm{B}$ and $\mathrm{F}$ subtypes: $\mathrm{B}_{\mathrm{PR}} \mathrm{B}_{\mathrm{RT}} \mathrm{B}_{\mathrm{gp} 120} \mathrm{~B}_{\mathrm{gp} 41}, \quad \mathrm{~F}_{\mathrm{PR}} \mathrm{F}_{\mathrm{RT}} \mathrm{F}_{\mathrm{gp} 120} \mathrm{~F}_{\mathrm{gp} 41}, \quad \mathrm{~F}_{\mathrm{PR}} \mathrm{F}_{\mathrm{RT}} \mathrm{B}_{\mathrm{gp} 120} \mathrm{~B}_{\mathrm{gp} 41}$, $\mathrm{F}_{\mathrm{PR}} \mathrm{B}_{\mathrm{RT}} \mathrm{B}_{\mathrm{gp} 120} \mathrm{~B}_{\mathrm{gp} 41}$, and $\mathrm{B}_{\mathrm{PR}} \mathrm{B}_{\mathrm{RT}} \mathrm{F}_{\mathrm{gp} 120} \mathrm{~B}_{\mathrm{gp} 41}$ (Table 1). When BED was performed with these plasma samples, 16 of 74 samples $(21.6 \%)$ yielded OD values suggestive of being recent HIV infection (seroconversion duration of 153 days).

Owing to the differences on the Stanford University Drug Resistance Database and IAS-USA Drug Resistance Mutations Web sites, RT and PR sequences were submitted to both algorithms to determine antiretroviral drug resistance mutations. What concerns to the PR accessory resistance mutations, L63P, and its substitutions T/A/C/ D/S/L/V/LP/PS (59.5\%), M36I (27\%), V77I (18.9\%), and I93L (17.6\%) were mostly observed. L63P and its substitutions occurred in 64.1 percent of B subtype samples and in 22.2 percent of the $\mathrm{F}$ samples whereas M36I was detected in 15.6 percent of the B samples and in all sub- type F samples. Both PR accessory resistance mutations occurred in the AGH mosaic. The K20R polymorphism was observed in only one sample of subtype F. Considering the two algorithms, no major PR resistance mutation was observed in the samples included in this study (Table 2).

For RT, both V106I and V118I, described as polymorphisms, were detected in four samples (5.5\%). Other polymorphisms (M41K, E44K, and K103R) not related to RT inhibitors were also detected. Two samples belonging to subtype B (2.7\%) showed evidence of NRTI resistance: the first with M41L and T215S primary mutations, confirmed by both algorithms, and a second one, with $\mathrm{T} 69 \mathrm{~N}$, that alone is capable of conferring potential low-level of resistance to D4T and DDI according to the Stanford Drug Resistance algorithm. Recombinant AGH and BC samples did not show any PR and RT resistance mutations (Table 2).

Resistance to the fusion inhibitor T20 was first determined based on previous reports ${ }^{38-40}$ taking into consideration the 32- to 69-amino-acid positions of the first heptad repeated (HR1) region of gp41. In this context, 8 of the 62 studied samples (12.9\%), all belonging to subtype $\mathrm{B}$, presented the following mutations: Q39R (1.6\%), L44M (1.6\%), R46M (1.6\%), and V69I (8.1\%). According to the IAS-USA Drug Resistance Mutation algorithm, however, only the Q39R (1.6\%) would be considered as associated with T20 resistance (Table 2). Other substitutions such as V38L, N42S, R46K, and L54M, not related to T20 resistance, were seen, respectively, in 1 (1.6\%), 1 (1.6\%), 4 (6.4\%), and 24 (39.3\%) of the analyzed samples.

\section{DISCUSSION}

As shown by the present work, subtype B is still the most frequent and disseminated HIV-1 subtype in Brazil, with the exception of the Southern region, ${ }^{6,12,15,16,18}$ contributing to approximately 84 percent of all cases, whereas subtype F counted only to 2.7 percent of the total of HIV-1 cases. Previous studies, ${ }^{8,10,16}$ based on unique genomic regions, have reported subtype $\mathrm{F}$ frequencies varying from 10 to 15.4 percent. In our study, analyzing PR, RT, and envelope (C2-V3 and gp41) genes, 10.8 percent of $\mathrm{BF}$ mosaics were evidenced, corroborating other reports. ${ }^{14,18,42}$ In Brazil, the simultaneous circulation of B and F subtypes in the Southeastern part of the country in association with increasing viral variability and recombination events would probably explain the occurrence of such $\mathrm{BF}$ mosaic frequency. The presence of a rare AGH variant sample is indicative of the emergence of new and different 


\begin{tabular}{|c|c|c|c|c|}
\hline Samples & $\begin{array}{l}\text { PR resistance } \\
\text { mutations }\end{array}$ & $\begin{array}{c}\text { NRTI resistance } \\
\text { mutations }\end{array}$ & $\begin{array}{c}\text { NNRTI resistance } \\
\text { mutations }\end{array}$ & $\begin{array}{l}\text { T20 resistance } \\
\text { mutations }\end{array}$ \\
\hline $1287-00$ & M36L & & & V69l \\
\hline $1292-00$ & L63P, V77I & $\mathrm{T} 6 \mathrm{~N}^{*}$ & & \\
\hline $258-01$ & M36I, L63T & V118I & & V69l \\
\hline 261-01 & M36I, I93L & & & Q39R* \\
\hline 575-01 & & & & V69I \\
\hline $908-01$ & L63P & & & V69l \\
\hline $1938-01$ & L63A & M41L, T215S* & & \\
\hline $2116-01$ & & & & V69l \\
\hline $2459-01$ & & & & R46M \\
\hline $1560-04$ & D60E, V77I & & & L44M \\
\hline \multicolumn{5}{|c|}{$\begin{array}{l}\text { * Major mutations are indicated. Sample } 1292-00 \text { was resistant to NRTI according to } \\
\text { Stanford University Drug Resistance Database; Sample 261-01 was resistant to T20 } \\
\text { according to IAS-USA Drug Resistance Mutations Algorithm; Sample 1938-01 was } \\
\text { resistant to NRTI according to both Stanford University and IAS-USA Drug Resistance } \\
\text { Mutations Algorithms. Other samples shown in this table were resistant to T20 according } \\
\text { to the criterion of other authors. }{ }^{38-40}\end{array}$} \\
\hline
\end{tabular}

not find PI, NRTI, and NNRTI primary resistance-associated mutations. Similar results were obtained in a French study, including 142 drug-naïve individuals from Cameroon, Senegal, Democratic Republic of Congo, and France, which did not reveal any primary resistance mutations either for PI or for NRTI. ${ }^{43}$

More recently, primary mutations related to PI, NNRTI, and NRTI in 2.24, 2.06, and 2.36 percent, respectively, have been reported among HIV-1infected drug-naïve population diagnosed at the Voluntary Counseling and Testing Centers located in different Brazilian major capitals. ${ }^{16}$ Except for the NNRTI and PI, NRTI drug resistancenon-B viral subtypes and reinforces the molecular HIV-1 diversity seen in the country for the past years. This fact is supported by two reports on the occurrence of a CRF 02_AG in Brazil. ${ }^{18,22}$

The lack of subtype $C$ in this study might be attributed to the absence of samples from the Southern region of the country, where such subtype is prevalent. ${ }^{14,16}$ Whereas several reports have described an increase in the frequency of subtype C over time in the Southern region, only sporadic cases of this subtype are being detected in other regions. One case of a BC mosaic genome, however, not previously observed in studies involving blood donors in the country, was detected in a sample from the northeastern region.

Resistance mutations associated to RT and PR in antiretroviral drug-naïve individuals might result from the transmission from an individual that has failed highly active antiretroviral therapy or be the consequence of divergent variants. Differences in the interpretation of the drug resistance mutation patterns were observed in the present study when with Stanford University Drug Resistance Database and IAS-USA current algorithms. According to both algorithms one sample displaying M41L and T215S primary mutations conferring resistance to abacavir, AZT, and D4T presented resistance to NRTI. A second sample showing a T69N mutation with a D4T resistance profile, however, was considered as NRTIresistant based in one of the algorithms. Major PR resistance mutations were not observed when applying either criterion.

In agreement with our findings, NNRTI-associated resistance mutations were not previously observed in drug-naïve HIV-1-infected blood donors, although 9.38 percent of the samples showed primary resistance to AZT in such study. ${ }^{26}$ In samples from blood donors collected in Brazil during the year 1999, other authors ${ }^{28}$ did associated mutation values were very close to those obtained in this study.

A significantly higher proportion (14\%) of virus presenting NRTI-related mutations was demonstrated in a study with 56 drug-naïve HIV-1-infected individuals from the Army Health Services in Rio de Janeiro, Brazil. ${ }^{22}$ Such findings could be suggestive of an increase in primary drug resistance in the country. Our results and those previously commented from various authors, however, confirm the considerably low levels of primary resistance in Brazil in comparison to those reported in other countries, ${ }^{29-33,44}$ varying from 10 to 26 percent.

The occurrence of 87.8 percent of PR secondary or accessory mutations, also documented in this study, was similar to what has been observed among blood donor samples collected in 1998 in Brazil. ${ }^{28}$ The most frequent PR polymorphisms observed were L63P and its substitutions and M36I. Both polymorphisms were also found in the AGH recombinant sample. All NRTI polymorphisms occurred in subtype B samples, the most common ones being V118I and V106I (5.5\%), in a higher frequency than observed by other authors. ${ }^{16,22}$

Differences in the interpretation of T20 fusion inhibitor primary resistance mutation patterns were also observed between the IAS-USA algorithm and a recent work from a Spanish group ${ }^{39}$ that considered other gp41 residues $(32,44,45,46$, and 69$)$ in addition to those (3643) previously defined. Taken together, only Q39R (1.6\%) was considered as a T20 resistance mutation in the current study. Other mutations such as L44M (1.6\%), R46M (1.6\%), and V69I $(8.1 \%)$ were also observed, however. Similar results were described in T20-naïve patients in Spain, with the evidence of Q39R, R46M, and V69I mutations in B and non-B subtypes. ${ }^{39}$ Another study reported no associated T20 resistance mutations among drug-naïve patients infected with B and non-B HIV-1 subtypes in the Luxembourg HIV cohort. ${ }^{38}$ Considering the high frequency of 
subtype B (83.8\%) in the present study, along with the limitation of non-B subtype diversity and the occurrence of all T20 resistance mutations in B samples, the influence of subtype under resistance selection to T20 remains unclear.

Finally, 21.6 percent of the analyzed samples corresponded to cases of recent infection as determined by the Calypte HIV-1 BED incidence EIA. Based on other authors' findings ${ }^{45}$ in a study with first-time and replacement blood donors, the residual risk of HIV transmission was found to be 14.9 per million units. In Brazil, that may represent at least 50 possible cases of infected blood transmissions among the 3.5 million donations per year in the country. The blood donors of the present project could be under the window period of the infection at the moment of a previous blood donation and not be detected by the current screening tests. These data emphasize the need to introduce nucleic acid-based assays in the screening of all blood to be transfused in the country given its capability of reducing the infection window period for HIV-1 in up to 7 and 9 to 13 days, respectively, compared to p24 and antibody detecting assays.

In this respect, the development of biologic panels of subtyped and characterized plasma samples will be of paramount importance to improve and expand the diagnosis of HIV-1 infection, adjusting the tests so as to consider all HIV-1 variants circulating in the country.

\section{ACKNOWLEDGMENTS}

We thank Monica Arruda (UFRJ) and Carlos Augusto Velasco de Castro (FIOCRUZ) for their special support on the laboratory activities.

\section{REFERENCES}

1. McCutchan FE, Salminen MO, Carr JK, Burke DS. HIV-1 genetic diversity. AIDS 1996;10(Suppl 3):S13-20.

2. Robertson DL, Anderson JP, Bradac JA, et al. HIV-1 nomenclature proposal. Science 2000;288:55-6.

3. Kandathil AJ, Ramalingam S, Kannangai R, David S, Sridharan G. Molecular epidemiology of HIV. Indian J Med Res 2005;121:333-44.

4. De Sa, Filho DJ, Sucupira MC, et al. Identification of two HIV type 1 circulating recombinant forms in Brazil. AIDS Res Hum Retroviruses 2006;22:1-13.

5. Epidemological Bulletin AIDS and STD Year II, no1-01st26th epidmological week January to June 2005.

6. Morgado MG, Sabino EC, Shpaer EG, et al. V3 region polymorphisms in HIV-1 from Brazil: prevalence of subtype B strains divergent from North American/European prototype and detection of subtype F. AIDS Res Hum Retroviruses 1994;10:569-76.

7. Louwagie J, Delwart EL, Mullins JI, et al. Genetic analysis of HIV-1 isolates from Brazil reveals presence of two distinct genetic subtypes. AIDS Res Hum Retroviruses 1994;10:561-7.

8. Morgado MG, Guimaraes ML, Gripp CB, et al. Molecular epidemiology of HIV-1 in Brazil: high prevalence of HIV-1 subtype B and identification of an HIV-1 subtype D infection in the city of Rio de Janeiro, Brazil. Evandro Chagas Hospital AIDS Clinical Research Group. J Acquir Immune Defic Syndr 1998;18:488-94.

9. Couto-Fernandez JC, Morgado MG, Bongertz V, et al. HIV-1 subtyping in Salvador, Bahia, Brazil: a city with African sociodemographic characteristics. J Acquir Immune Defic Syndr 1999;22:288-93.

10. Tanuri A, Swanson P, Devare S, et al. HIV-1 subtypes among blood donors from Rio de Janeiro, Brazil. J Acquir Immun Defic Human Retrovirol 1999;20:60-6.

11. Tanuri A, Vicente AC, Otsuki K, et al. Genetic variation and susceptibilities to protease inhibitors among subtype B and F isolates in Brazil. Antimicrob Agents Chemother 1999;43: 253-8.

12. Caride E, Brindeiro R, Hertogs $\mathrm{K}$, et al. Drug-resistance reverse transcriptase genotyping and phenotyping of $\mathrm{B}$ and non-B subtypes ( $F$ and A) of human immunodeficiency virus type 1 found in Brazilian patients failing HAART. Virology 2000;275:107-15.

13. Stefani MM, Pereira GA, Martelli CM, Shindo N, GalvaoCastro B. Evidence of HIV-1 genetic diversity among pregnant women with AIDS or infected with HIV-1 in Central Brazil. J Acquir Immune Defic Syndr 2000;2: 205-7.

14. Guimarães ML, Santos Moreira A, Loureiro R, Galvao-Castro B, Morgado MG. Brazilian network for HIV isolation and characterization: high frequency of recombinant genomes in HIV type 1 samples from Brazilian southeastern and southern regions. AIDS Res Hum Retroviruses 2002;18: 1261-9.

15. Morgado MG, Guimaraes ML, Galvao-Castro B. HIV-1 polymorphism: a challenge for vaccine development-a review. Mem Inst Oswaldo Cruz 2002;97:143-50.

16. Brindeiro RM, Diaz RS, Sabino EC, et al. Brazilian Network for Drug Resistance Surveillance (HIV BResNet): a survey of chronically infected individuals. AIDS 2003;17:1063-9.

17. Soares MA, Oliveira T, Brindeiro RM, et al. A specific subtype $\mathrm{C}$ of human immunodeficiency vírus type 1 circulates in Brazil. AIDS 2003;17:11-21.

18. Couto-Fernandez JC, Silva-de-Jesus C, Veloso VG, et al. Human immunodeficiency virus type 1 (HIV-1) genotyping in Rio de Janeiro, Brazil: assessing subtype and drugresistance associated mutations in HIV-1 infected individuals failing highly active antiretroviral therapy. Mem Inst Oswaldo Cruz 2005;100:73-8.

19. Potts KE, Kalish ML, Lott T, et al. Genetic heterogeneity of the V3 region of the HIV-1 envelope glycoprotein in Brazil. AIDS 1993;7:1191-7.

20. Covas DT, Biscaro TA, Kashima S, Duarte G, Machado AA. High frequency of the GWG (Pro Trp) envelope variant of 
HIV-1 in Southeast Brazil. J Acquir Immune Defic Syndr Hum Retrovirol 1998;19:74-9.

21. Sabino E, Shpaer E, Morgado MG, et al. Identification of an HIV-1 proviral genome recombinant between subtype B and $\mathrm{F}$ in PBMCs obtained from an individual in Brazil. JVirol 1994;68:6340-6.

22. Pires IL, Soares MA, Speranza FA, et al. Prevalence of human immunodeficiency virus drug resistance mutations and subtypes in drug-naive, infected individuals in the army health service of Rio de Janeiro, Brazil. J Clin Microbiol 2004;42:426-30.

23. Simon F, Ly TD, Baillou-Beaufils A, et al. Sensitivity of screening kits for anti-HIV-1 subtype O antibodies. AIDS 1994;8:1628-9.

24. Apetrei C, Loussert-Ajaka I, Descamps D, et al. Lack of screening test sensitivity during HIV-1 non subtype B seroconversion. AIDS 1996;10:F57-60.

25. De Oliveira CF, Diaz RS, Machado DM, et al. Surveillance of HIV-1 genetic subtypes and diversity in the US blood supply. Transfusion 2000;40:1399-406.

26. Brindeiro R, Vanderborght B, Caride E, et al. Sequence diversity of the reverse transcriptase of human immunodeficiency virus type 1 from untreated Brazilian individuals. Antimicrob Agents Chemother 1999;43:1674-80.

27. Kupek EM. HIV seroprevalence among blood donors in southern Brazil in the decade of 1990. Braz J Infect Dis 2000;4:217-25.

28. Dumans AT, Soares MA, Pieniazek D, et al. Prevalence of protease and reverse transcriptase drug resistance mutations over time in drug-naive human immunodeficiency virus type 1-positive individuals in Rio de Janeiro, Brazil. Antimicrob Agents Chemother 2002;46:3075-9.

29. Little SJ, Holte S, Routy JP, et al. Antiretroviral-drug resistance among patients recently infected with HIV. N Engl J Med 2002;347:385-94.

30. Tamalet C, Pasquier C, Yahi N, et al. Prevalence of drug resistant mutants and virological response to combination therapy in patients with primary HIV-1 infection. J Med Virol 2000;61:181-6.

31. Chaix ML, Descamps D, Harzic M, et al. Stable prevalence of genotypic drug resistance mutations but increase in non-B virus among patients with primary HIV-1 infection in France. AIDS 2003;17:2635-43.

32. Duwe S, Brunn M, Altmann D, et al. Frequency of genotypic and phenotypic drug-resistant HIV-1 among therapy-naive patients of the German Seroconverter Study. J Acquir Immune Defic Syndr 2001;26:266-73.

33. Briones C, Perez-Olmeda M, Rodríguez C, et al. Primary genotypic and phenotypic HIV-1 drug resistance in recent seroconverters in Madrid. J Acquir Immune Defic Syndr 2001;26:145-50.

34. Magiorkinis E, Paraskevis D, Magiorkinis G, et al. Mutations associated with genotypic resistance to anti-retroviral therapy in treatment naive HIV-1 infected patients in Greece. Virus Res 2002;85:109-15.

35. Afani A, Ayala M, Meyer A, Cabrera R, Acevedo W. Primary resistance to antiretroviral therapy in patients with HIV/ AIDS in Chile. Rev Med Chil 2005;133:295-301.

36. Kantor R, Katzenstein DA, Efron B, et al. Impact of HIV-1 subtype and antiretroviral therapy on protease and reverse transcriptase genotype: results of a global collaboration. PLoS Med 2005;2:e112.

37. Xu L, Hue S, Taylor S, et al. Minimal variation in T-20 binding domain of different HIV-1 subtypes from antiretroviralnaive and -experienced patients. AIDS 2002;16:1684-6.

38. Roman F, Gonzalez D, Lambert C, et al. Uncommon mutations at residue positions critical for enfuvirtide (T-20) resistance in enfuvirtide-naive patients infected with subtype B and non-B HIV-1 strains. J Acquir Immune Defic Syndr 2003;33:134-9.

39. Carmona R, Perez-Alvarez L, Munoz M, et al. Natural resistance-associated mutations to Enfuvirtide (T20) and polymorphisms in the gp41 region of different HIV-1 genetic forms from T20 naive patients. J Clin Virol 2005;32:248-53.

40. Aghokeng AF, Ewane L, Awazi B, et al. Enfuvirtide binding domain is highly conserved in non-B HIV type 1 strains from Cameroon, West Central Africa. AIDS Res Hum Retroviruses 2005;21:430-3.

41. Parekh BS, Dougal JS. New approaches for detecting recent HIV-1 infection. AIDS Rev 2001;3:183-93.

42. De Sá Filho DJ, Sanabani S, Diaz RS, et al. Analysis of fulllength human immunodeficiency virus type 1 genome reveals a variable spectrum of subtypes B and $\mathrm{F}$ recombinants in Sao Paulo, Brazil. AIDS Res Hum Retroviruses 2005;21:145-51.

43. Vergne L, Peeters M, Mpoudi-Ngole E, et al. Genetic diversity of protease and reverse trancriptase sequences in nonsubtype-B human immunodeficiency virus type 1 strains: evidence of many minor drug resistance mutations in treatment-naïve patients. J Clin Microbiol 2000;38:3919-25.

44. Puig T, Perez-Olmeda M, Rubio A, et al. Prevalence of genotypic resistance to nucleoside analogues and protease inhibitors in Spain. The ERASE-2 Study Group. AIDS 2000;14:727-32.

45. Barreto CC, Sabino EC, Goncalez TT, et al. Prevalence, incidence, and residual risk of human immunodeficiency virus among community and replacement first-time blood donors in Sao Paulo, Brazil. Transfusion 2005;45: 1709-14. 